\title{
Multicentre study of hospitalised patients with sports- and recreational cycling-related traumatic brain injury in Hong Kong
}

Peter YM Woo *, Eric Cheung, Fion WY Lau, Nancy WS Law, Carly KY Mak, Peony Tan, Bertrand Siu, Anson Wong, Calvin HK Mak, KY Chan, KY Yam, KY Pang, YC Po, WM Lui, Danny TM Chan, WS Poon

\section{A B S T R A C T}

Introduction: Cycling is associated with a greater risk of traumatic brain injury (TBI) than other recreational activities. This study aimed to investigate the epidemiology of sports-related TBI in Hong Kong and to examine predictors for recreational cycling-induced intracranial haemorrhage.

Methods: This retrospective multicentre study included patients diagnosed with sports-related TBI in public hospitals in Hong Kong from 2015 to 2019. Computed tomography scans were reviewed by an independent assessor. The primary endpoint was traumatic intracranial haemorrhage. The secondary endpoint was an unfavourable Glasgow Outcome Scale (GOS) score at discharge from hospital.

Results: In total, 720 patients were hospitalised with sports-related TBI. The most common sport was cycling (59.2\%). The crude incidence of cycling-related TBI was 1.1 per 100000 population. Cyclists were more likely to exhibit intracranial haemorrhage and an unfavourable GOS score, compared with patients who had TBI because of other sports. Although 47\% of cyclists had intracranial haemorrhage, only $15 \%$ wore a helmet. In multivariate analysis, significant predictors for intracranial haemorrhage were age $\geq 60$ years, antiplatelet medication, moderate or severe TBI, and skull fracture. Among 426 cyclists, 375 (88\%) had mild TBI, and helmet wearing was protective against intracranial haemorrhage, regardless of age, antiplatelet medication intake, and mechanism of injury. Of 426 cyclists, 31 (7.3\%) had unfavourable outcomes on discharge from hospital.

Conclusions: The incidence of sports-related TBI is low in Hong Kong. Although cycling-related head injuries carried greater risks of intracranial haemorrhage and unfavourable outcomes compared with other sports, most cyclists experienced good recovery. Helmet wearing among recreational cyclists with mild TBI was protective against intracranial haemorrhage and skull fracture.

Hong Kong Med J 2021;27:338-49

https://doi.org/10.12809/hkmj208934

Department of Neurosurgery, Kwong Wah Hospital, Hong Kong

Department of Neurosurgery, Queen Elizabeth Hospital, Hong Kong

Department of Neurosurgery, Tuen Mun Hospital, Hong Kong

Department of Neurosurgery, Pamela Youde Nethersole Eastern Hospital, Hong Kong

Department of Neurosurgery, Princess Margaret Hospital, Hong Kong

Division of Neurosurgery, Department of Surgery, Queen Mary Hospital, Hong Kong

Division of Neurosurgery, Department of Surgery, Prince of Wales Hospital, Hong Kong

* Corresponding author: wym307@ha.org.hk

New knowledge added by this study

- The incidence of sports-related traumatic brain injury (TBI) is lower in Hong Kong than in other countries or regions; cycling is the sport most frequently associated with TBI.

- A greater proportion of hospitalised patients with cycling-related TBI had intracranial haemorrhage and unfavourable functional outcomes, compared with patients who had TBI because of other sports. Risk factors for intracranial haemorrhage were older age ( $>60$ years), antiplatelet medication intake, moderate or severe TBI, and skull fracture.

- Only $15 \%$ of hospitalised patients with cycling-related TBI wore a helmet at the time of injury; none of the patients who died had been wearing a helmet.

- The lack of an independent association with motor vehicle collisions suggests that recreational cycling at comparatively low speeds without protective head gear can be fatal.

Implications for clinical practice or policy

- Cycling is becoming increasingly popular, but Hong Kong is one of the most dangerous regions in the world for cyclists in terms of fatality rate.

- Public health policies that improve bicycle rider safety (eg, mandatory helmet legislation) should be deliberated. Although helmet wearing is protective against intracranial haemorrhage for mild TBI individuals, the rate of its adoption is low.

- Measures to control the risk of sports-related TBI should be carefully considered when designing public health policies to promote sports engagement. 


\section{Introduction}

Considerable physical and psychosocial benefits are associated with participation in sporting activities. ${ }^{1,2}$ Physical activity has been demonstrated to reduce the risks of coronary heart disease, some cancers, obesity, hypertension, and type 2 diabetes mellitus..$^{2-6}$ Its obvious merits have prompted several national health programmes, including the health programme in Hong Kong, to promote sports to the general public. $^{7-9}$ However, sports participation carries a risk of injury, especially traumatic brain injury (TBI). It has been estimated that $20 \%$ of all TBIs are sportsrelated. ${ }^{10}$ In addition, up to $20 \%$ of sports-related TBI survivors (usually adolescents or young adults) experience chronic symptoms including headache, fatigue, and cognitive and balance difficulties. ${ }^{11}$ There is a global trend of increasing sports-related TBI incidence: from 3.5 to 31.5 per 100000 population in the past decade. ${ }^{12,13}$ Because many patients with mild TBI do not seek medical attention, these figures likely underestimate the total burden of this condition. ${ }^{13}$ Population-based studies reviewing sports-related TBI are sparse; most target specific groups of individuals (eg, professional athletes) or rely on self-reporting surveys that lack a uniform definition and comprehensive assessment of brain injury. ${ }^{14}$ For similar reasons, studies reviewing outcome predictors have also been inadequate, thus hindering the generation of meaningful conclusions to guide governmental policy initiatives. ${ }^{14}$

Hong Kong is highly urbanised with an established public transport system, such that cycling is mainly regarded as a recreational activity. ${ }^{15,16}$ In terms of fatality rate, Hong Kong is among the most dangerous areas for cycling, compared with other cities such as New York, the US, or countries such as France. $^{17}$

This study was performed to document the epidemiology of sports-related TBI among patients who required inpatient care by adopting territorywide uniform diagnostic coding criteria, clear data definitions, and systematic assessments of radiologic findings. Because cycling is a popular sport in Hong Kong, factors predictive of intracranial haemorrhage (eg, the effect of helmet use) and poor functional outcomes among cyclists hospitalised with TBI were determined.

\section{Methods}

Patients who required inpatient care at any Hospital Authority institution for sports-related TBI from 1 January 2015 to 31 December 2019 were reviewed. The Hospital Authority is a public health service highly subsidised by the Hong Kong SAR Government; it is responsible for $90 \%$ of inpatient bed days in the city. Patients were identified by the International Classification of Diseases, Tenth

\section{與運動及休閒式騎車相關創傷性腦損傷住院患者 的香港多中心研究}

\author{
胡日明、張旭康、劉穎瑜、羅詠詩、麥嘉怡、陳施蓓、 \\ 蕭浚鏗、王思晴、麥凱鈞、陳廣有、任廣銳、彭佳源、 \\ 保延聰、呂偉文、陳達明、潘偉生
}

引言：與其他休閒活動相比, 騎車造成創傷性腦損傷（TBI）的風險

較高。本研究旨在確定香港與運動相關TBI的流行病學和因騎車造成 顱內出血的預測因素。

方法：這項回顧性多中心研究對象為2015年至2019年於公立醫院住 院的因運動引致TBI的患者, 並由獨立評估員審查患者的電腦掃描結 果。研究的主要終點是創傷性顱內出血。次要終點是出院時不利的格 拉斯哥昏迷指數。

結果 : 納入 720 名因運動引致TBI的住院患者, 當中最普遍原因為騎 車 $(59.2 \%)$, 其粗發病率為 $1.1 / 100000$ 。與其他運動引致TBI的患 者相比, 騎車者較易出現顱內出血和不利的昏迷指數。研究群組中騎 車者有㲊內出血佔 $47 \%$, 但當中只有 $15 \%$ 佩戴頭盔。多變量分析顯示 騎車者出現創傷性顱內出血的顯著因素包括60歲或以上、服用抗血小 板藥物、中度或重度TBI及顱骨骨折。在 426 名因騎車引致TBI的患者 中, 375 名有輕度TBI; 佩戴頭盔能減低顱內出血的機會, 這與年齡、 抗血小板藥物攝入和損傷機制並無相關。在 426 名因騎車引致TBI的患 者中, 只有 31 名 $(7.3 \%)$ 在出院時有不利結果。

結論 : 在香港, 與運動相關的TBI發病率較低。儘管與其他運動相 比, 頭部受傷的騎車者發生臚內出血和不利結果的風險較高, 但大部 份在體能上都能有良好的恢復。騎車造成輕度TBI的住院患者因事發 時佩戴頭盔, 可減低顱內出血和覤頁骨骨折的機會。

Revision, Clinical Modification code (ICD-10-CM) designation for TBI 854.0 secondary to a sportsrelated external cause (E codes: E006-10). Data from clinical records, operation notes, medication prescriptions, and computed tomography (CT) brain scans from a central digital imaging repository were reviewed. In particular, the type of sport played, the clinical presentation of symptoms, the Injury Severity Score (ISS), the need for neurosurgery, length of hospitalisation, and diagnosis of postconcussion syndrome were recorded. Head injury was classified into mild (presenting Glasgow Coma Scale [GCS] score, 14-15); moderate (presenting GCS score, 9-13), and severe (presenting GCS score, 3-8), in accordance with criteria established by the Neurotraumatology Committee of the World Federation of Neurosurgical Societies. ${ }^{18}$ Postconcussion syndrome was defined in accordance with ICD-10 criteria. This required a 4-week duration of symptoms from at least three categories following a traumatic loss of consciousness. The symptom categories were headache, irritability, concentration impairment, insomnia, and a preoccupation with the aforementioned symptoms. For neurosurgical patients with cycling related-TBI, the mechanism of injury and their experience level (ie, professional athlete or amateur rider) were documented. All CT 
scans were reviewed by an independent assessor with 6 months of neurosurgical training experience who was blinded to the patients' clinical characteristics and outcomes. The scans were first evaluated using the Rotterdam CT score, a commonly utilised validated radiological assessment system for the prognosis of patients with TBI. The classification has four distinct elements that require the appraisal of the degree of basal cistern obliteration, degree of midline shift, the presence (or absence) of an epidural mass lesion, and the presence (or absence) of intraventricular or traumatic subarachnoid haemorrhage (Table 1). In addition, the scans were assessed for skull fractures, cerebral contusions, and acute subdural haematomas (ASDHs). The primary outcome of the study was the presence of intracranial haemorrhage on the admitting $\mathrm{CT}$ scan. All potential predictors were categorised into patient-related, trauma-related, and radiological factors. The secondary outcome was unfavourable functional performance, defined as a Glasgow Outcome Scale (GOS) score of 3 to 5 on discharge from the hospital (3, severe disability; 4, persistent vegetative state; and 5, death).

Statistical analyses utilised the Chi squared test and Fisher's exact test were used for categorical data such as patient gender or the use of antiplatelet medication. Independent-samples $t$ test was used for continuous data such as patient age or duration of hospitalisation. Multivariate binary logistic regression was used to identify independent factors for the presence (or absence) of traumatic intracranial haemorrhage. A P value of $<0.05$ was considered statistically significant. Statistical analysis was conducted using SPSS (Windows version 20.0; IBM Corp, Armonk [NY], US).

TABLE I. Rotterdam CT scores for traumatic brain injury

\begin{tabular}{lc}
\hline Predictor & Score \\
\hline Basal cisterns & 0 \\
\hline Normal & 1 \\
Compressed & 2 \\
\hline Absent & \\
Midline shift & 0 \\
\hline No or $\leq 5$ mm & 1 \\
\hline Shift $>5$ mm & \\
\hline Epidural mass lesion & 0 \\
\hline Present & 1 \\
\hline Absent & \\
\hline Intraventricular blood or tSAH & 0 \\
\hline Absent & 1 \\
\hline Present & +1 \\
\hline Sum score & \\
\hline
\end{tabular}

Abbreviations: $\mathrm{CT}=$ computed tomography; $\mathrm{tSAH}=$ traumatic subarachnoid haemorrhage

\section{Results}

Overall characteristics of patients with sports-related traumatic brain injury during the study period

In total, 720 consecutive patients were hospitalised with sports-related TBI during the 5-year study period, and 705 (97.9\%) of them were admitted under neurosurgical care. This was equivalent to a crude incidence of 1.9 per 100000 general population. The mean ( \pm standard deviation $[S D]$ ) age was $32 \pm 19$ years; 521 (72.4\%) patients were adults ( $\geq 18$ years) and 568 (78.9\%) were male. The most common sport was cycling $(59.2 \%)$, followed by football $(21.3 \%)$ and basketball $(7.5 \%)$ [Fig a]. On admission, most (86.1\%) patients were fully conscious. Overall, 658 (91.4\%) patients had mild TBI, $41(5.7 \%)$ patients had moderate TBI, and 21 (2.9\%) patients had severe TBI. Post-traumatic seizures occurred in 36 (5.0\%) patients. Furthermore, $324(45.0 \%)$ patients had a loss of consciousness and 269 (37.4\%) patients experienced post-traumatic retrograde amnesia. Only 19 (2.6\%) patients were taking either antiplatelet or anticoagulant medication. Extracranial injuries were sustained by 208 (28.9\%) patients; among them, injuries were mainly either limb abrasions or contusions (62.1\%). The median ISS was 2 (interquartile range $=2-8$ ).

Intracranial haemorrhage was noted in 283 (39.3\%) patients with TBI; 166 (23.1\%) patients exhibited traumatic subarachnoid haemorrhage and 157 (21.8\%) exhibited ASDH. Skull fractures were detected in 179 (24.9\%) patients, with a median Rotterdam CT score of 2 (interquartile range $=2-2$ ). In total, $59(8.2 \%)$ patients required neurosurgical intervention; 32 (54.2\%) of them had good recovery with a median GOS score of 5 on discharge from the hospital and at 6 months. The mean ( \pm SD) duration of hospitalisation was $5 \pm 28$ days. Among 307 (42.6\%) patients in whom 6-month GOS scores could be assessed, good recovery was observed in 260 (84.7\%). Post-concussion syndrome was diagnosed in 30 (6.2\%) of 482 patients who attended scheduled follow-up outpatient consultations.

\section{Recreational cycling-related traumatic brain injury}

The crude incidence of recreational cycling-related TBI requiring hospitalisation was 1.1 per 100000 population. A comparison was performed between cyclists with sports-related TBI and patients who had TBI because of other sports (Table 2). Cyclists were significantly older $(\mathrm{P}<0.001)$ [Table 2 ; Fig b]. Among 426 cyclists, 306 (71.8\%) were male and 120 (28.2\%) were female. However, the proportion of patients who were female was significantly higher among those who had TBI because of cycling (28.2\%) than among those who had TBI because of other sports 
(10.9\%; $\mathrm{P}<0.001)$. Cyclists were likely to exhibit more severe TBI with an almost three-fold greater risk of sustaining extracranial injury (odds ratio [OR] 2.8; 95\% CI: 1.9-4.0), resulting in a higher ISS $(\mathrm{P}<0.001)$. Of cyclists admitted for head injury, 201 (47.2\%) had intracranial haemorrhage, which was radiologically more extensive in terms of the Rotterdam CT score, compared with haemorrhage in non-cyclists $(\mathrm{P}<0.01)$. As a consequence, a greater proportion of cyclists had a worse GOS score on discharge from hospital (OR 2.8; 95\% CI: 1.3-6.2) and at 6 months (OR 4.7; 95\% CI: 2.1-10.5). The cause of death for all cyclists with 30-day mortality was severe TBI with medically refractory intracranial hypertension. Although the overall incidence was low, cyclists also had a greater risk of post-concussion syndrome (OR 2.5; 95\% CI: 1.2-5.4).

\section{Predictors for traumatic intracranial haemorrhage and poor functional outcome at discharge from hospital among cyclists}

Among the 426 cyclists in this study, 128 (30.0\%) experienced bicycle accidents during the weekend; $10(2.3 \%)$ of the cyclists were professional athletes. Most cyclists $(273 ; 64.1 \%)$ accidently fell off their bicycle on their own (ie, without colliding into another object) on level ground. Of the injuries, $103(24.2 \%)$ were sustained when the cyclist was traveling downhill; for the 28 patients with records of self-reported velocities, the estimated mean $( \pm \mathrm{SD})$ velocity at the moment before injury was $40 \pm 15 \mathrm{~km} / \mathrm{h}$. At the time of injury, 361 (84.7\%) cyclists had not been wearing a helmet. Among eight (1.9\%) patients who subsequently died, none had been wearing protective head gear.

Risk factors for traumatic intracranial haemorrhage among cyclists are shown in Table 3. Univariate analysis identified the following risk factors: age $\geq 60$ years, use of antiplatelet medication, involvement in a motor vehicle collision, presence of moderate to severe TBI, and skull fracture. In univariate analysis, helmet wearing was protective against intracranial haemorrhage. Multivariate logistic regression identified the following independent risk factors: age $\geq 60$ years, antiplatelet medication intake, moderate or severe TBI, and the presence of a skull fracture. Table 4 shows independent significant predictors for unfavourable GOS score on discharge from hospital: age $\geq 60$ years, antiplatelet intake, severe TBI, intracranial haemorrhage, and the need for neurosurgical operative intervention.

\section{Effect of helmet use among cyclists}

As shown in Table 2, 375 (88.0\%) hospitalised cyclists had mild TBI, whereas only $36(8.5 \%)$ cyclists had moderate TBI and 15 (3.5\%) had severe TBI. No

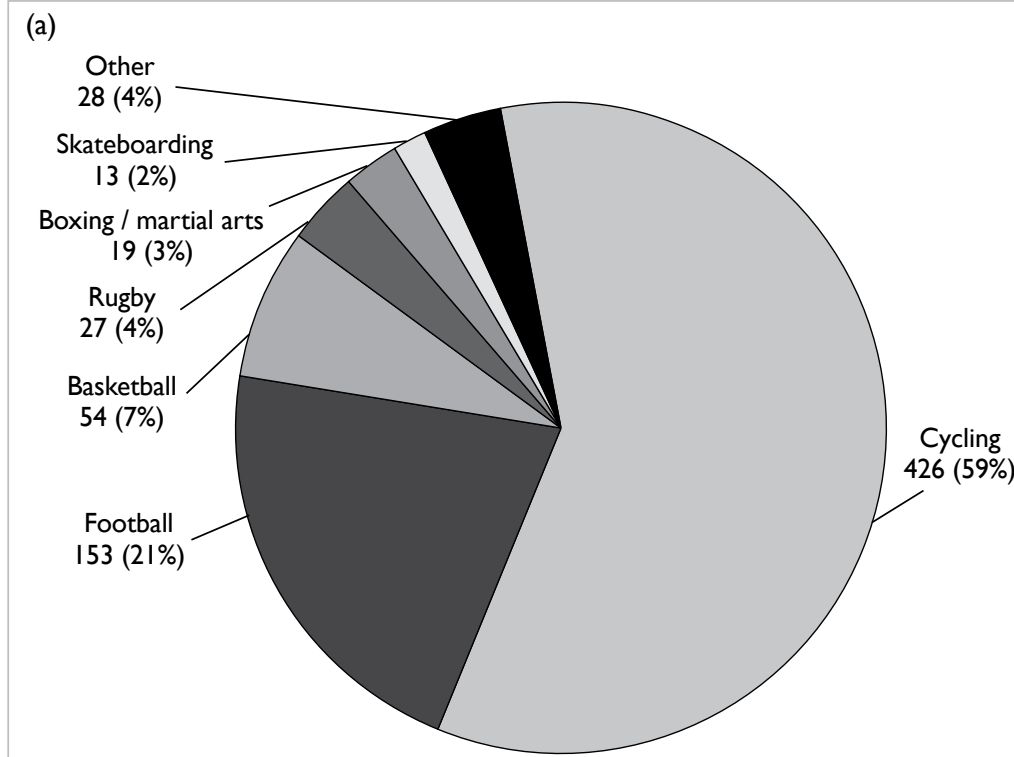

(b)

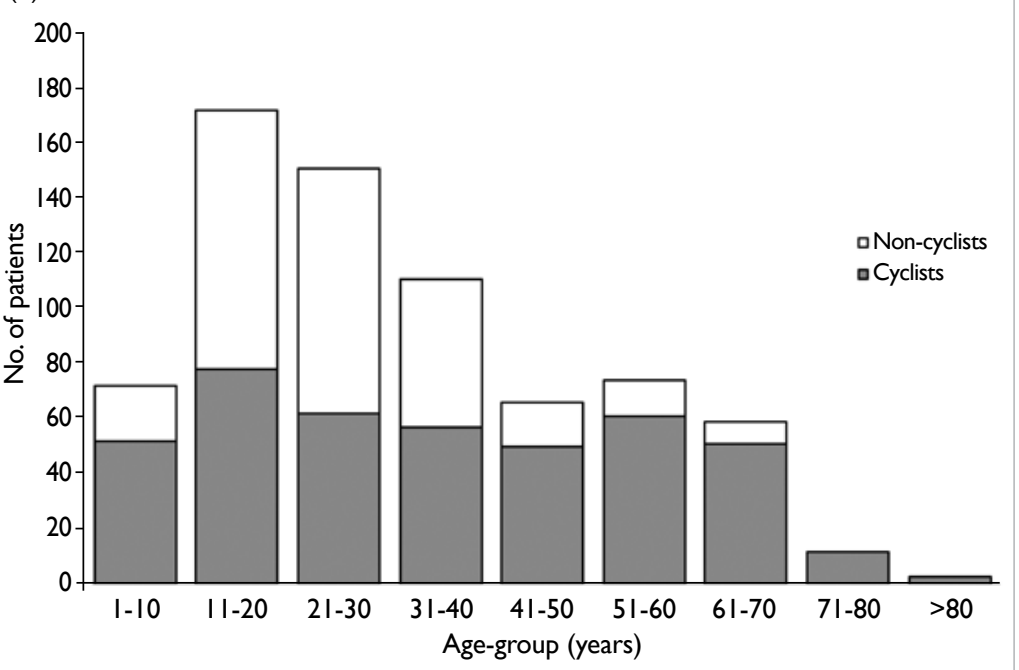

FIG. (a) Sports played among 720 hospitalised patients diagnosed with sportsrelated traumatic brain injury from 2015 to 2019 . (b) Histogram depicting the number of patients with head injury because of cycling (grey bars) compared with those who had head injury because of other sports (white bars), according to age-group

protective effect of helmet use was noted in terms of reducing TBI severity across these GCS-defined categories (Table 3). However, among cyclists with mild TBI, helmets were significantly protective against intracranial haemorrhage and skull fracture, regardless of age, antiplatelet medication intake, or mechanism of injury (Table 5). Although the median Rotterdam CT score was comparable between cyclists with mild TBI who did or did not wear helmets $(P=0.68)$, significantly fewer patients with head protection had epidural haematoma or ASDH. For patients with mild TBI who had intracranial haemorrhage, this difference in radiological factors 
TABLE 2. Comparison of baseline characteristics and outcomes between cyclists and non-cyclists with sports-related traumatic brain injury"

\begin{tabular}{|c|c|c|c|}
\hline & Non-cyclist $(n=294)$ & Cyclist $(n=426)$ & $\begin{array}{c}\text { OR (95\% } \mathrm{Cl}) \text { or } \\
\mathrm{P} \text { value }\end{array}$ \\
\hline Age, $y$, mean $\pm S D$ & $26 \pm 13$ & $35 \pm 21$ & $<0.001$ \\
\hline Age $\geq 60$ y & $10(3.4 \%)$ & $71(16.7 \%)$ & $5.7(2.9-11.2)$ \\
\hline Male sex & $262(89.1 \%)$ & $306(71.8 \%)$ & $0.3(0.2-0.5)$ \\
\hline Antiplatelet medication & $5(1.7 \%)$ & $9(2.1 \%)$ & NS \\
\hline Anticoagulant medication & $2(0.7 \%)$ & $3(0.7 \%)$ & NS \\
\hline \multicolumn{4}{|l|}{ Trauma factors } \\
\hline \multicolumn{4}{|l|}{ TBI severity } \\
\hline Mild & $283(96.3 \%)$ & $375(88.0 \%)$ & $0.29(0.1-0.6)$ \\
\hline Moderate & $5(1.7 \%)$ & $36(8.5 \%)$ & $5.4(2.1-13.8)$ \\
\hline Severe & $6(2.0 \%)$ & $15(3.5 \%)$ & NS \\
\hline Post-traumatic amnesia & $96(32.7 \%)$ & $173(40.6 \%)$ & $1.4(1.0-1.9)$ \\
\hline Post-traumatic seizures & $26(8.8 \%)$ & $10(2.3 \%)$ & $0.2(0.1-0.5)$ \\
\hline Loss of consciousness & $111(37.8 \%)$ & $213(50.0 \%)$ & $1.7(1.2-2.3)$ \\
\hline Associated extracranial injury & $51(17.3 \%)$ & $157(36.9 \%)$ & $2.8(1.9-4.0)$ \\
\hline ISS, median (IQR) & $2(2-8)$ & $3(2-9)$ & $<0.001$ \\
\hline ISS $\geq 3$ & $40(13.6 \%)$ & $123(28.9 \%)$ & $2.6(1.7-3.8)$ \\
\hline Skull fracture & $59(20.1 \%)$ & $120(28.2 \%)$ & $1.6(1.1-2.2)$ \\
\hline Intracranial haemorrhage & $82(27.9 \%)$ & $201(47.2 \%)$ & $2.3(1.7-3.2)$ \\
\hline $\mathrm{EDH}$ & $14(4.8 \%)$ & $30(7.0 \%)$ & NS \\
\hline ASDH & $54(18.4 \%)$ & $103(24.2 \%)$ & NS \\
\hline Traumatic SAH & $40(13.6 \%)$ & $126(29.6 \%)$ & $2.7(1.8-4.0)$ \\
\hline Cerebral contusion & $25(8.5 \%)$ & $81(19.0 \%)$ & $2.5(1.6-4.1)$ \\
\hline Rotterdam CT score, mean \pm SD & $2.2 \pm 0.6$ & $2.3 \pm 0.7$ & $<0.01$ \\
\hline Rotterdam CT score $\geq 3$ & $40(13.6 \%)$ & $123(28.9 \%)$ & $2.6(1.7-3.8)$ \\
\hline Need for neurosurgery & $24(8.2 \%)$ & $35(8.2 \%)$ & NS \\
\hline Hospitalisation duration, $d$, mean $\pm S D$ & $4 \pm 13$ & $6 \pm 34$ & NS \\
\hline 30-Day mortality & $4(1.4 \%)$ & $8(1.9 \%)$ & NS \\
\hline Unfavourable GOS score ${ }^{\dagger}$ at discharge from hospital & $8(2.7 \%)$ & $31(7.3 \%)$ & $2.8(1.3-6.2)$ \\
\hline Unfavourable GOS score at 6 months ${ }^{\ddagger}$ & $8(2.6 \%)$ & $39(12.7 \%)$ & $4.7(2.1-10.5)$ \\
\hline Post-concussion syndrome & $11(3.7 \%)$ & $28(6.6 \%)$ & $2.5(1.2-5.4)$ \\
\hline
\end{tabular}

Abbreviations: $95 \% \mathrm{Cl}=95 \%$ confidence interval; $\mathrm{ASDH}=$ acute subdural haematoma; $\mathrm{CT}$ = computed tomography; $\mathrm{EDH}=$ epidural haematoma; GOS = Glasgow Outcome Scale; IQR = interquartile range; ISS = Injury Severity Score; NS = not significant; $\mathrm{OR}=$ odds ratio; $\mathrm{SAH}=$ subarachnoid haemorrhage; $\mathrm{SD}=$ standard deviation; $\mathrm{TBI}$ = traumatic brain injury

* Data are shown as No. (\%), unless otherwise specified

Unfavourable GOS score defined as 3-5 (3: severe disability, 4: persistent vegetative state; 5 : death)

307 Patients attended follow-up consultations at 6 months

led to a significantly shorter mean $( \pm \mathrm{SD})$ duration of hospitalisation for patients who wore helmets (2.6 \pm 2.9 days), compared with patients who did not (7.1 \pm 11.6 days, $\mathrm{P}<0.001)$. However, there was no difference in the need for neurosurgical intervention among patients with mild TBI who had intracranial haemorrhage according to head protection status $(\mathrm{P}=0.17)$. Similarly, unfavourable GOS scores on discharge from hospital $(\mathrm{P}=0.43)$ and at 6 months $(\mathrm{P}=0.71)$ were comparable among patients with mild
TBI who had intracranial haemorrhage, regardless of head protection status (Table 5 ).

\section{Discussion}

\section{Balancing sports engagement with sports- related traumatic brain injury}

The incidence of sports-related TBI in Hong Kong is 2 per 100000 general population; this is lower than in other countries (eg, the US, Australia, or 
TABLE 3. Predictors for traumatic intracranial haemorrhage among cyclists*

\begin{tabular}{|c|c|c|c|c|}
\hline & $\begin{array}{c}\text { No intracranial } \\
\text { haemorrhage } \\
(n=225)\end{array}$ & $\begin{array}{c}\text { Intracranial } \\
\text { haemorrhage } \\
(n=201)\end{array}$ & $\begin{array}{l}\text { Univariate OR } \\
\text { (95\% Cl) or } \\
\text { P value }\end{array}$ & $\begin{array}{c}\text { Adjusted OR } \\
\text { (95\% Cl) }\end{array}$ \\
\hline \multicolumn{5}{|l|}{ Patient factors } \\
\hline Age, $y$, mean $\pm S D$ & $30 \pm 19$ & $42 \pm 21$ & $<0.001$ & \\
\hline Age $\geq 60 y$ & $25(11.1 \%)$ & $46(22.9 \%)$ & $2.4(1.4-4.0)$ & $1.7(1.0-3.1)$ \\
\hline Male sex & $168(74.7 \%)$ & $138(68.7 \%)$ & NS & \\
\hline Antiplatelet medication & 0 & $8(4.0 \%)$ & $2.2(2.0-2.4)$ & $2.0(1.8-2.2)$ \\
\hline Anticoagulant medication & $1(0.4 \%)$ & $1(0.5 \%)$ & NS & \\
\hline Professional athlete & $4(1.8 \%)$ & $6(3.0 \%)$ & NS & \\
\hline \multicolumn{5}{|l|}{ Trauma factors } \\
\hline \multicolumn{5}{|l|}{ Mechanism of injury } \\
\hline MVA & $4(1.8 \%)$ & $13(6.5 \%)$ & $4.0(1.3-12.4)$ & \\
\hline Lost balance on level ground & $105(46.7 \%)$ & $76(37.8 \%)$ & NS & \\
\hline Fell on downhill slope & $53(23.6 \%)$ & $50(24.9 \%)$ & NS & \\
\hline Collided with stationary object & $19(8.4 \%)$ & $12(6.0 \%)$ & NS & \\
\hline Collided with another cyclist & $17(7.6 \%)$ & $13(6.5 \%)$ & NS & \\
\hline \multicolumn{5}{|l|}{ TBI severity } \\
\hline Mild (reference) & - & - & & \\
\hline Moderate & $3(1.3 \%)$ & $33(16.4 \%)$ & $14.5(4.4-48.2)$ & $11.2(3.2-40.0)$ \\
\hline Severe & $2(0.9 \%)$ & $13(6.5 \%)$ & $7.7(1.7-34.4)$ & $7.9(1.4-66.7)$ \\
\hline Helmet wearing & $42(18.7 \%)$ & $23(11.4 \%)$ & $0.6(0.3-0.9)$ & \\
\hline TBI during the weekend & $64(28.4 \%)$ & $64(31.8 \%)$ & NS & \\
\hline Post-traumatic amnesia & $96(42.7 \%)$ & $77(38.3 \%)$ & NS & \\
\hline Post-traumatic seizures & $3(1.3 \%)$ & $7(3.5 \%)$ & NS & \\
\hline Loss of consciousness & $111(49.3 \%)$ & $102(50.7 \%)$ & NS & \\
\hline Vomiting & $32(14.2 \%)$ & $38(18.9 \%)$ & NS & \\
\hline Skull fracture & $39(17.3 \%)$ & $81(40.3 \%)$ & $3.2(2.1-5.0)$ & $2.7(1.6-4.5)$ \\
\hline
\end{tabular}

Abbreviations: 95\% Cl = 95\% confidence interval; MVA = motor vehicle accident; NS = not significant; OR = odds ratio; SD = standard deviation; $\mathrm{TBI}=$ traumatic brain injury

* Data are shown as No. (\%), unless otherwise specified

Italy), where the incidences range from 4 to 32 per 100000 population. ${ }^{12}$ The lower incidence in Hong Kong is consistent with a previous finding that Hong Kong residents (especially children and adolescents) have lower physical activity and fitness levels than in other regions, according to a global evidence-based evaluation of such indicators from 49 countries. ${ }^{19}$ Considering the health benefits of an active lifestyle, there is a clear need to promote sports engagement in Hong Kong. A survey of 5701 residents performed by the Transport Department of the Hong Kong SAR Government estimated that $10 \%$ of households had bicycles available for use; moreover, $69 \%$ (4 million) of residents aged 15 years or older knew how to ride one. ${ }^{16}$ In addition, most survey respondents (73\%) cycled for recreational or fitness purposes. ${ }^{15}$

\section{Cycling safety outcomes in Hong Kong}

Previous epidemiological studies of sports-related brain injuries revealed that cycling was one of the most frequent activities involved. ${ }^{10,12,20,21}$ In 2019, 1738 road traffic accidents involving cyclists were reported to the Hong Kong Transport Department. ${ }^{22}$ Half of these accidents (50.6\%, 879/1738) occurred in recreational areas such as cycling tracks, parks, or playgrounds; eight $(0.5 \%)$ patients experienced fatal injuries. ${ }^{22}$ In the past 10 years, the number of cyclist injuries in Hong Kong has increased by $5.2 \%$ per year. ${ }^{17}$ Compared with other regions worldwide, Hong Kong is one of the most dangerous areas for cycling. ${ }^{17}$ The fatality rate (per billion minutes cycled) in the city was 34 , substantially higher than the rates in Stockholm, Sweden (3), France (4), and 
TABLE 4. Predictors for unfavourable GOS score at discharge from hospital (severe disability, vegetative state, or death) among cyclists with $\mathrm{TBI}^{*}$

\begin{tabular}{|c|c|c|c|c|}
\hline & $\begin{array}{l}\text { Favourable GOS } \\
\text { score }(n=395)\end{array}$ & $\begin{array}{l}\text { Unfavourable GOS } \\
\text { score }(n=31)\end{array}$ & $\begin{array}{l}\text { Univariate OR } \\
\text { (95\% Cl) or } \\
\mathrm{P} \text { value }\end{array}$ & $\begin{array}{l}\text { Adjusted OR } \\
(95 \% \mathrm{Cl})\end{array}$ \\
\hline \multicolumn{5}{|l|}{ Patient factors } \\
\hline Age, $y$, mean $\pm S D$ & $34 \pm 20$ & $55 \pm 19$ & $<0.001$ & \\
\hline Age $\geq 60 y$ & $60(15.2 \%)$ & $11(35.5 \%)$ & $3.0(1.4-6.7)$ & $12.0(1.8-20.1)$ \\
\hline Male sex & $284(71.9 \%)$ & $22(71.0 \%)$ & NS & \\
\hline Antiplatelet medication & $4(1.0 \%)$ & $4(12.9 \%)$ & $14.3(3.4-60.1)$ & $17.9(2.0-33.3)$ \\
\hline Anticoagulant medication & $2(0.5 \%)$ & 0 & NS & \\
\hline Professional athlete & $10(2.5 \%)$ & 0 & NS & \\
\hline \multicolumn{5}{|l|}{ Trauma factors } \\
\hline MVA & $13(3.3 \%)$ & $4(12.9 \%)$ & $4.5(1.3-14.9)$ & \\
\hline \multicolumn{5}{|l|}{ TBI severity } \\
\hline Mild (reference) & - & & & \\
\hline Moderate & $24(6.1 \%)$ & $12(38.7 \%)$ & $9.8(4.2-22.4)$ & \\
\hline Severe & $3(0.8 \%)$ & $12(38.7 \%)$ & $82.3(21.4-316.4)$ & $50.0(1.7-91.0)$ \\
\hline Head protection worn & $61(15.4 \%)$ & $4(12.9 \%)$ & NS & \\
\hline Post-traumatic seizures & $10(2.5 \%)$ & 0 & NS & \\
\hline Associated extracranial injury & $143(36.2 \%)$ & $14(45.2 \%)$ & NS & \\
\hline ISS, median (IQR) & $3(2-8)$ & $33(8-50)$ & $<0.001$ & \\
\hline ISS $\geq 3$ & $267(67.6 \%)$ & $30(96.8 \%)$ & $14.3(1.9-28.4)$ & \\
\hline \multicolumn{5}{|l|}{ Radiological factors } \\
\hline Intracranial haemorrhage & $173(43.8 \%)$ & 28 (90.3\%) & $12.0(3.6-40.1)$ & $11.6(3.23-35.7)$ \\
\hline $\mathrm{EDH}$ & $25(6.3 \%)$ & $5(16.1 \%)$ & $2.8(1.0-8.0)$ & \\
\hline ASDH & $80(20.3 \%)$ & $23(74.2 \%)$ & $11.3(4.9-26.3)$ & \\
\hline Traumatic SAH & $101(25.6 \%)$ & $25(80.6 \%)$ & $12.1(4.8-30.4)$ & \\
\hline Cerebral contusion & $59(14.9 \%)$ & $22(71.0 \%)$ & $13.9(6.1-31.7)$ & \\
\hline Rotterdam CT score, median (IQR) & $2(2-2)$ & $3(2-4)$ & $<0.001$ & \\
\hline Rotterdam CT score $\geq 3$ & $96(24.3 \%)$ & $27(87.1 \%)$ & $21.0(7.2-61.6)$ & \\
\hline Need for neurosurgery & $15(3.8 \%)$ & 20 (64.5\%) & $46.1(18.8-113.1)$ & $12.7(1.3-121.0)$ \\
\hline
\end{tabular}

Abbreviations: $95 \% \mathrm{Cl}=95 \%$ confidence interval; $\mathrm{ASDH}=$ acute subdural haematoma; $\mathrm{CT}$ = computed tomography; $\mathrm{EDH}=$ epidural haematoma; GOS = Glasgow Outcome Scale; IQR = interquartile range; ISS = Injury Severity Score; MVA = motor vehicle accident; NS = not significant; $\mathrm{OR}$ = odds ratio; $\mathrm{SAH}=$ subarachnoid haemorrhage; $\mathrm{SD}=$ standard deviation; $\mathrm{TBI}=$ traumatic brain injury

* Data are shown as No. (\%), unless otherwise specified

other metropolitan areas (eg, New York City [18] and Los Angeles [8]). ${ }^{17}$ These studies included riders primarily involved in commuting and the causes of death were not elucidated, but they indicate a growing need to enhance the safety of vulnerable road users.

To our knowledge, this is the first multicentre study to comprehensively document the outcomes of inpatients with recreational cycling-related TBI using standard assessment criteria. By comparison with patients who had TBI because of other sports, we found that cyclists in Hong Kong exhibited greater risks of more severe injury, intracranial haemorrhage, unfavourable GOS score at discharge from hospital, and post-concussion syndrome. Despite these findings, our results suggest that cycling is generally safe and hospitalised patients had a high (92.7\%) likelihood of favourable functional outcomes on discharge from hospital.

Single-centre reviews of cycling-related injuries among various suburban districts in Hong Kong found that limb injuries were the most common form of trauma followed by head injury (10\%-39\% of patients) ${ }^{23-26}$ Among patients with TBI, 
TABLE 5. Comparison of baseline characteristics and outcomes between helmet-wearing and non-helmet wearing cyclists hospitalised with mild TBI*

\begin{tabular}{|c|c|c|c|}
\hline & $\begin{array}{l}\text { Not wearing helmet } \\
(n=297)\end{array}$ & $\begin{array}{l}\text { Wearing helmet } \\
(n=56)\end{array}$ & OR (95\% Cl) \\
\hline \multicolumn{4}{|l|}{ Patient factors } \\
\hline Age, $y$, mean $\pm S D$ & $33 \pm 21$ & $36 \pm 18$ & NS \\
\hline Age $\geq 60 y$ & 47 (15.8\%) & $9(16.1 \%)$ & NS \\
\hline Male sex & $204(68.7 \%)$ & 47 (83.9\%) & $2.4(1.1-5.1)$ \\
\hline Antiplatelet medication & $6(2.0 \%)$ & 0 & NS \\
\hline Anticoagulant medication & $1(0.3 \%)$ & $1(1.8 \%)$ & NS \\
\hline Professional athlete & $2(0.7 \%)$ & $6(10.7 \%)$ & $17.7(3.5-90.2)$ \\
\hline \multicolumn{4}{|l|}{ Trauma factors } \\
\hline \multicolumn{4}{|l|}{ Mechanism of injury } \\
\hline MVA & $10(3.4 \%)$ & $2(3.6 \%)$ & NS \\
\hline Lost balance on level ground & $204(68.7 \%)$ & $35(62.5 \%)$ & NS \\
\hline Fell on downhill slope & $64(21.5 \%)$ & $12(21.4 \%)$ & NS \\
\hline Collided with stationary object & $23(7.7 \%)$ & $2(3.6 \%)$ & NS \\
\hline Collided with another cyclist & $15(5.1 \%)$ & $5(8.9 \%)$ & NS \\
\hline Associated extracranial injury & $102(34.3 \%)$ & $23(41.1 \%)$ & NS \\
\hline ISS, median (IQR) & $30(21-41)$ & $34(8-51)$ & NS \\
\hline ISS $\geq 3$ & $44(14.8 \%)$ & $10(17.9 \%)$ & NS \\
\hline \multicolumn{4}{|l|}{ Radiological factors } \\
\hline Skull fracture & $75(25.3 \%)$ & $7(12.5 \%)$ & $0.4(0.2-0.9)$ \\
\hline Intracranial haemorrhage & $128(43.1 \%)$ & $15(26.8 \%)$ & $0.5(0.3-0.9)$ \\
\hline EDH & $20(6.7 \%)$ & 0 & $0.8(0.7-0.9)$ \\
\hline ASDH & $63(21.2 \%)$ & $4(7.1 \%)$ & $0.3(0.1-0.8)$ \\
\hline Traumatic SAH & $71(23.9 \%)$ & $9(16.1 \%)$ & NS \\
\hline Cerebral contusion & $40(13.5 \%)$ & $6(10.7 \%)$ & NS \\
\hline Rotterdam CT score, mean \pm SD & $2(2-2)$ & $2(2-2)$ & NS \\
\hline Rotterdam CT score $\geq 3$ & $67(22.6 \%)$ & $9(16.1 \%)$ & NS \\
\hline Need for neurosurgery & $6(2.0 \%)$ & $2(3.6 \%)$ & NS \\
\hline Hospitalisation duration, $d$, mean \pm SD & $2 \pm 3$ & $3 \pm 6$ & NS \\
\hline 30-Day mortality & 0 & 0 & NS \\
\hline Unfavourable GOS score ${ }^{\dagger}$ at discharge from hospital & $6(2.0 \%)$ & 0 & NS \\
\hline Unfavourable GOS score at 6 months ${ }^{\ddagger}$ & $16(5.4 \%)$ & $2(3.6 \%)$ & NS \\
\hline Post-concussion syndrome & $6(2.0 \%)$ & $2(3.6 \%)$ & NS \\
\hline
\end{tabular}

Abbreviations: 95\% Cl = 95\% confidence interval; $\mathrm{ASDH}=$ acute subdural haematoma; $\mathrm{CT}$ = computed tomography; $\mathrm{EDH}=$ epidural haematoma; GOS = Glasgow Outcome Scale; IQR = interquartile range; ISS = Injury Severity Score; MVA = motor vehicle accident; $\mathrm{NS}$ = not significant; $\mathrm{OR}=$ odds ratio; $\mathrm{SAH}=$ subarachnoid haemorrhage; $\mathrm{SD}=$ standard deviation; $\mathrm{TBI}=$ traumatic brain injury

Data are shown as No. (\%), unless otherwise specified

Unfavourable GOS score defined as 3-5 (3: severe disability, 4: persistent vegetative state; 5: death)

₹ 126 Patients attended follow-up consultations at 6 months

$16 \%$ to $53 \%$ exhibited "severe" injury; however, the had moderate to severe TBI. There was also a high studies did not provide explicit definitions to qualify incidence of intracranial haemorrhage involving this categorisation, and did not describe radiological almost half of the patients. Both these factors were data regarding the extent of injury or the need independent predictors of poor GOS score on for neurosurgical intervention. ${ }^{23,26}$ In the present discharge from hospital. Our results are consistent study, $12 \%$ of hospitalised cyclists with head injury with the findings in a previous study where $75 \%$ 
of all cycling-related deaths were caused by severe TBI. ${ }^{27}$ The lack of an independent association with motor vehicle collisions, which constituted only a minority of injuries in this cohort, suggests that recreational cycling at comparatively low speeds can be fatal. Notably, the mechanism of injury for four (50\%) of the eight recreational cyclists who died in this study was a loss of balance, followed by a fall on level ground without colliding into another object, person, or motor vehicle.

\section{Helmet use: safety and legislative implications}

Previous studies in Hong Kong, the most recent of which was performed $>10$ years ago, revealed that recreational cyclists rarely wore protective headgear (eg, frequencies of $0.2 \%$ to $2.2 \%$ among emergency department attendees) ${ }^{24-26}$ Our findings revealed that significantly more patients (15\%) wore helmets at the time of injury. Governmental advocacy initiatives for promoting helmet wearing in recent years may have resulted in heightened public awareness regarding the risks of head trauma. ${ }^{28}$ There is little doubt that helmets are protective. In the past 30 years, several case-control and epidemiological studies have delivered compelling evidence to support the efficacy of bicycle helmet wearing in reducing the risk of life-threatening $\mathrm{TBI}^{29-37}$ In a case-control prospective multicentre study of over 3000 patients, Thompson et $\mathrm{al}^{33}$ noted that helmets (irrespective of design) conferred up to a $74 \%$ reduction in TBI during accidents. A subsequent meta-analysis of five studies observed that helmets provided a $63 \%$ to $88 \%$ reduction in the risk of head, brain, and severe TBI for all ages of cyclists; this included equal levels of protection for collisions involving motor vehicles and collisions due to other causes. ${ }^{38}$ In the present study, helmet wearing did not reduce TBI severity according to our broadly predefined categories. However, among hospitalised recreational cyclists with mild head injury, helmets did provide significant protection against intracranial haemorrhage, including potentially life-threatening epidural haematomas and ASDHs, as well as skull fractures. Thus, our findings may have important public health implications with regard to introducing mandatory bicycle helmet wearing legislation in the city.

Whether such laws should exist is a particularly divisive issue among public health experts and interest groups. ${ }^{39-43}$ In Australia, a nation with all-age helmet wearing safety laws, an overall $46 \%$ decline in cyclist fatalities per 1000000 population has been reported, compared with the pre-legislation period. ${ }^{44}$ Similar findings were noted in New Zealand: a $67 \%$ decline in severe TBI was recorded after the introduction of helmet laws.$^{45}$ In the US, a significant reduction in paediatric cyclist fatalities involving motor vehicles was observed in states with such laws. ${ }^{46}$ A meta-analysis of the effectiveness of bicycle helmet legislation revealed that it increased helmet usage, while significantly reducing head injuries and mortality. ${ }^{47}$ Several medical associations have expressed support for introducing such legislation; these include the World Health Organization, the British Medical Association, the American Medical Association, and the Royal Australasian College of Surgeons. ${ }^{48-51}$ However, critics of such compulsory policies have hypothesised that helmets could encourage risk-compensation behaviour, whereby cyclists may be more willing to engage in potentially injurious risks or for motorists to exercise less caution when encountering them..$^{39,52,53}$ Other reasons for opposition include infringement on individual liberties; some public health scholars have theorised that such laws could discourage cyclists from participating in gainful physical activity. ${ }^{39-42,54}$ From a Hong Kong Transport Department survey (5701 respondents) regarding attitudes towards possible helmet law and enforcement measures, the majority of respondents (78\%-90\%) were in favour of introducing such legislation, especially when riding on carriageways. ${ }^{16}$ However, among respondents who knew how to ride a bicycle (3933 respondents), $23 \%$ declared they would ride less frequently if mandatory helmet wearing was required. ${ }^{16}$

\section{Limitations}

An inherent limitation of a retrospective study of this nature was the likely under-reporting of the number of patients with sports-related head injuries. In the only existing population-based study of TBI epidemiology that included community-based injuries, 95\% were considered mild and $28 \%$ of respondents did not seek medical attention. ${ }^{55}$ Among professional or university-level athletes, under-reporting is more apparent: questionnaire surveys reveal that $31 \%$ to $78 \%$ of respondents neglected to pursue medical care despite experiencing a concussion during the preceding 12 months. ${ }^{56,57}$ At the emergency department level, no territory-wide TBI registry exists in Hong Kong; moreover, diagnostic coding to facilitate data retrieval is typically not performed after consultations. Therefore, we could only identify hospitalised patients with sports-related TBI by means of an administrative database that utilised the ICD-10 coding system. However, the validity of such administrative data for research has been questioned.$^{58}$ Studies have shown significantly lower TBI rates among young adults, men, and patients with less severe injuries when the ICD system was utilised, compared with thorough medical record review. ${ }^{59}$ Analysis of a population-based TBI sample showed that only $19 \%$ of individuals were assigned a TBI-related diagnostic ICD code. ${ }^{60}$ In addition, a degree of selection bias may have existed because some non-hospitalised helmet-wearing cyclists with 
mild head injury may have been discharged from the emergency department, mitigating the protective effects of helmet use. This may explain why no considerable differences in outcomes were detected for patients with moderate or severely injured patients. Despite the low rate of helmet use among recreational cyclists (15\%), significant protective effects were detected among mildly injured patients with regard to intracranial haemorrhage and skull fracture. This limited participant identification approach also allowed for a pragmatic review of patients with clinically significant TBI who were hospitalised following evaluation by an emergency care physician. Computed tomography scans are generally performed only for hospitalised patients with head injury in our public healthcare system; this approach offered an opportunity to evaluate imaging data for intracranial haemorrhage. Because the ICD coding system for traumatic intracranial haemorrhage reportedly has high sensitivity and specificity (both $>80 \%$ ), 59 we adopted this coding outcome as the study's primary endpoint. Another important limitation was the definition of mild TBI, which affected most patients in this study. The definitions offered by several authorities range from conventional GCS-based criteria such as the US Centers for Disease Control and Prevention, ${ }^{61}$ and the American College of Surgeons ${ }^{62}$ to additional symptoms of confusion, memory impairment, transient loss of consciousness, and irritability proposed by the World Health Organisation and the American Congress of Rehabilitation Medicine. ${ }^{18,63,64}$ A better delineation of these symptoms would have enhanced the identification of patients with "highrisk" mild TBI; however, because these relevant symptoms were often not systematically documented in most medical records retrieved in our study, we used GCS-based criteria to reduce the overall rate of underdiagnosis. Using GOS score on discharge from hospital as a secondary study endpoint, we found that only $31(7 \%)$ patients had unfavourable outcomes. Although statistically significant predictors for TBI were identified, the wide confidence intervals for these predictors suggest that the sample size was insufficient to draw robust conclusions. Finally, we could only retrospectively assess GOS score as a fundamental measure of functional outcome. More sensitive instruments (eg, the extended GOS or the Sport Concussion Assessment Tool $^{65,66}$ ) might have been better for assessing the psychosocial and cognitive aspects of TBI, considering that a large proportion of mildly injured cyclists had intracranial haemorrhage.

\section{Conclusions}

The incidence of sports-related TBI in Hong Kong is low and cycling is the most frequently associated activity. Almost half of hospitalised recreational cyclists sustained intracranial haemorrhage. Compared with patients who had head injury because of other sports, cyclists are more likely to experience severe consequences. There is evidence that helmet use offers protection against intracranial haemorrhage and skull fracture among cyclists with mild head injury. Cycling is a safe physical activity, but further legislative measures should be introduced to promote and protect the welfare of individuals enjoying this sport.

\section{Author contributions}

Concept or design: PYM Woo, E Cheung.

Acquisition of data: PYM Woo, E Cheung, FWY Lau, NWS Law, CKY Mak, P Tan, B Siu, A Wong.

Analysis or interpretation of data: PYM Woo, E Cheung, CKY Mak.

Drafting of the manuscript: All authors.

Critical revision of the manuscript for important intellectual content: All authors.

All authors had full access to the data, contributed to the study, approved the final version for publication, and take responsibility for its accuracy and integrity.

\section{Conflicts of interest}

All authors have disclosed no conflicts of interest.

\section{Declaration}

This research has not been presented or published in any form prior to submission.

\section{Funding/support}

This research received no specific grant from any funding agency in the public, commercial, or not-for-profit sectors.

\section{Ethics approval}

This study was approved by the Kowloon Central Cluster/ Kowloon East Cluster research ethics committee (Ref KCC/ KEC-2020-0331). All patients were treated in accordance with the Declaration of Helsinki. Informed consent was obtained from either the patient, next-of-kin, or their legal guardian.

\section{References}

1. Allender S, Cowburn G, Foster C. Understanding participation in sport and physical activity among children and adults: a review of qualitative studies. Health Educ Res 2006;21:826-35.

2. Warburton DE, Nicol CW, Bredin SS. Health benefits of physical activity: the evidence. CMAJ 2006;174:801-9.

3. Batty D, Thune I. Does physical activity prevent cancer? Evidence suggests protection against colon cancer and probably breast cancer. BMJ 2000;321:1424-5.

4. Batty GD, Lee IM. Physical activity and coronary heart disease. BMJ 2004;328:1089-90.

5. Aune D, Norat T, Leitzmann M, Tonstad S, Vatten LJ. Physical activity and the risk of type 2 diabetes: a systematic review and dose-response meta-analysis. Eur J Epidemiol 2015;30:529-42.

6. Liu X, Zhang D, Liu Y, et al. Dose-response association between physical activity and incident hypertension: a 
systematic review and meta-analysis of cohort studies. Hypertension 2017;69:813-20.

7. Malm C, Jakobsson J, Isaksson A. Physical activity and sports-real health benefits: a review with insight into the Public Health of Sweden. Sports (Basel) 2019;7:127.

8. Puckett M, Neri A, Underwood JM, Stewart SL. Nutrition and physical activity strategies for cancer prevention in current national comprehensive cancer control program plans. J Community Health 2016;41:1013-20.

9. Department of Health, Hong Kong SAR Government. Towards 2025: strategy and action plan to prevent and control non-communicable diseases in Hong Kong. 2018 Available from: https://www.chp.gov.hk/files/pdf/ saptowards2025_fullreport_en.pdf. Accessed 2 Aug 2020.

10. Theadom A, Starkey NJ, Dowell T, et al. Sports-related brain injury in the general population: an epidemiological study. J Sci Med Sport 2014;17:591-6.

11. Manley G, Gardner AJ, Schneider KJ, et al. A systematic review of potential long-term effects of sport-related concussion. Br J Sports Med 2017;51:969-77.

12. Theadom A, Mahon S, Hume P, et al. Incidence of sportsrelated traumatic brain injury of all severities: a systematic review. Neuroepidemiology 2020;54:192-9.

13. Daneshvar DH, Nowinski CJ, McKee AC, Cantu RC. The epidemiology of sport-related concussion. Clin Sports Med 2011;30:1-17.

14. Selassie AW, Wilson DA, Pickelsimer EE, Voronca DC Williams NR, Edwards JC. Incidence of sport-related traumatic brain injury and risk factors of severity: a population-based epidemiologic study. Ann Epidemiol 2013;23:750-6.

15. Transport Department, Hong Kong SAR Government. Cycling study. 2004. Available from: https://www.td.gov hk/filemanager/en/publication/cyclingstudy.pdf. Accessed 22 Aug 2020.

16. Transport Department. Hong Kong SAR Government. Travel Characteristics Survey 2011 Final Report. Available from: https://www.td.gov.hk/filemanager/en/ content_4652/tcs2011_eng.pdf. Accessed 22 Aug 2020.

17. Xu P, Dong N, Wong SC, Huang H. Cyclists injured in traffic crashes in Hong Kong: a call for action. PLoS One 2019;14:e0220785.

18. Servadei F, Teasdale G, Merry G, Neurotraumatology Committee of the World Federation of Neurosurgical Societies. Defining acute mild head injury in adults: a proposal based on prognostic factors, diagnosis, and management. J Neurotrauma 2001;18:657-64.

19. Huang WY, Wong SH, Sit CH, et al. Results from the Hong Kong's 2018 report card on physical activity for children and youth. J Exerc Sci Fit 2019;17:14-9.

20. Harris AW, Jones CA, Rowe BH, Voaklander DC. A population-based study of sport and recreation-related head injuries treated in a Canadian health region. J Sci Med Sport 2012;15:298-304.

21. Beck AJ, Kee J. The epidemiology of sports head injuries in a rural population. Br J Sports Med 2011;45:A20.

22. Transport Department, Hong Kong SAR Government. Road Traffic Accident Statistics. Year 2019. Road traffic accidents involving bicycle by location and severity 2019. Available from: https://www.td.gov.hk/en/road safety/road_traffic_accident_statistics/2019/index.html. Accessed 9 Aug 2020.

23. Lee LL, Yeung KL, Chan JT, Chen RC. A profile of bicycle- related injuries in Tai Po. Hong Kong J Emerg Med 2003;10:81-7.

24. Yeung JH, Leung CS, Poon WS, Cheung NK, Graham CA, Rainer TH. Bicycle related injuries presenting to a trauma centre in Hong Kong. Injury 2009;40:555-9.

25. Ng CP, Siu AY, Chung CH. Bicycle-related injuries: a local scene. Hong Kong J Emerg Med 2001;8:78-83.

26. Sze NN, Tsui KL, Wong SC, So FL. Bicycle-related crashes in Hong Kong: is it possible to reduce mortality and severe injury in the metropolitan area? Hong Kong J Emerg Med 2011;18:136-43.

27. Rivara FP, Thompson DC, Thompson RS. Epidemiology of bicycle injuries and risk factors for serious injury. Inj Prev 1997;3:110-4.

28. Transport Department, Hong Kong SAR Government. Be a smart cyclist. Wear bicycle helmet always. 2020. Available from: https://www.td.gov.hk/filemanager/en/ content_4551/221201047_leaflet_a.pdf. Accessed 21 Aug 2020.

29. Attewell RG, Glase K, McFadden M. Bicycle helmet efficacy: a meta-analysis. Accid Anal Prev 2001;33:345-52.

30. Maimaris C, Summers CL, Browning C, Palmer CR. Injury patterns in cyclists attending an accident and emergency department: a comparison of helmet wearers and nonwearers. BMJ 1994;308:1537-40.

31. McDermott FT, Lane JC, Brazenor GA, Debney EA. The effectiveness of bicyclist helmets: a study of 1710 casualties. J Trauma 1993;34:834-44.

32. Thomas S, Acton C, Nixon J, Battistutta D, Pitt WR, Clark R. Effectiveness of bicycle helmets in preventing head injury in children: case-control study. BMJ 1994;308:173-6.

33. Thompson DC, Rivara FP, Thompson RS. Effectiveness of bicycle safety helmets in preventing head injuries. A casecontrol study. JAMA 1996;276:1968-73.

34. Thompson RS, Rivara FP, Thompson DC. A case-control study of the effectiveness of bicycle safety helmets. N Engl J Med 1989;320:1361-7.

35. Strotmeyer SJ, Behr C, Fabio A, Gaines BA. Bike helmets prevent pediatric head injury in serious bicycle crashes with motor vehicles. Inj Epidemiol 2020;7(Suppl 1):24.

36. Sethi M, Heidenberg J, Wall SP, et al. Bicycle helmets are highly protective against traumatic brain injury within a dense urban setting. Injury 2015;46:2483-90.

37. Persaud N, Coleman E, Zwolakowski D, Lauwers B, Cass D. Nonuse of bicycle helmets and risk of fatal head injury: a proportional mortality, case-control study. CMAJ 2012;184:E921-3.

38. Thompson DC, Rivara FP, Thompson R. Helmets for preventing head and facial injuries in bicyclists. Cochrane Database Syst Rev 2000;1999(2):CD001855.

39. Robinson DL. No clear evidence from countries that have enforced the wearing of helmets. BMJ 2006;332:722-5.

40. Hong Kong Cycling Alliance. Helmets. Available from: http://hkcyclingalliance.org/on-the-road/helmets. Accessed 21 Aug 2020.

41. Bateman-House A. Bikes, helmets, and public health: decision-making when goods collide. Am J Public Health 2014;104:986-92.

42. Goldacre B, Spiegelhalter D. Bicycle helmets and the law. BM) 2013;346:f3817.

43. Rivara FP, Thompson DC, Patterson MQ, Thompson RS. Prevention of bicycle-related injuries: helmets, education, and legislation. Annu Rev Public Health 1998;19:293-318. 
44. Olivier J, Boufous S, Grzebieta R. The impact of bicycle helmet legislation on cycling fatalities in Australia. Int J Epidemiol 2019;48:1197-203.

45. Tin Tin S, Woodward A, Ameratunga S. Injuries to pedal cyclists on New Zealand roads, 1988-2007. BMC Public Health 2010;10:655.

46. Meehan WP 3rd, Lee LK, Fischer CM, Mannix RC. Bicycle helmet laws are associated with a lower fatality rate from bicycle-motor vehicle collisions. J Pediatr 2013;163:726-9.

47. Macpherson A, Spinks A. Bicycle helmet legislation for the uptake of helmet use and prevention of head injuries. Cochrane Database Syst Rev 2008;2008(3):CD005401.

48. World Health Organisation. Why are helmets needed? 2006. Available from: https://www.who.int/roadsafety/ projects/manuals/helmet_manual/1-Why.pdf. Accessed 13 Oct 2021.

49. British Medical Association. Healthy transport=healthy lives. 2012. Available from: https://thepep.unece.org/ sites/default/files/2017-06/Healthy\%20transport\%20 healthy\%20lives\%20Britsh\%20Medical\%20Association. pdf. Accessed 13 Oct 2021.

50. American Medical Association. Helmets for riders of motorized and non-motorized cycles H-10.964. Available from: https://policysearch.ama-assn.org/policyfinder/ detail/bicycle helmets?uri=\%2FAMADoc\%2FHOD. xml-0-3.xml. Accessed 20 Sep 2021.

51. McDermott FT. Helmet efficacy in the prevention of bicyclist head injuries: Royal Australasian College of Surgeons initiatives in the introduction of compulsory safety helmet wearing in Victoria, Australia. World J Surg 1992;16:379-83.

52. Gamble T, Walker I. Wearing a bicycle helmet can increase risk taking and sensation seeking in adults. Psychol Sci 2016;27:289-94.

53. Walker I. Drivers overtaking bicyclists: objective data on the effects of riding position, helmet use, vehicle type and apparent gender. Accid Anal Prev 2007;39:417-25.

54. Hooper C, Spicer J. Liberty or death; don't tread on me. J Med Ethics 2012;38:338-41.

55. Theadom A, Barker-Collo S, Feigin VL, et al. The spectrum captured: a methodological approach to studying incidence and outcomes of traumatic brain injury on a population level. Neuroepidemiology 2012;38:18-29.
56. Delaney JS, Lamfookon C, Bloom GA, Al-Kashmiri A, Correa JA. Why university athletes choose not to reveal their concussion symptoms during a practice or game. Clin J Sport Med 2015;25:113-25.

57. Meehan WP 3rd, Mannix RC, O'Brien MJ, Collins MW. The prevalence of undiagnosed concussions in athletes. Clin J Sport Med 2013;23:339-42.

58. van Walraven C, Bennett C, Forster AJ. Administrative database research infrequently used validated diagnostic or procedural codes. J Clin Epidemiol 2011;64:1054-9.

59. Carroll CP, Cochran JA, Guse CE, Wang MC. Are we underestimating the burden of traumatic brain injury? Surveillance of severe traumatic brain injury using Centers for Disease Control International Classification of Disease, Ninth Revision, Clinical Modification, traumatic brain injury codes. Neurosurgery 2012;71:1064-70.

60. Barker-Collo S, Theadom A, Jones K, Feigin VL, Kahan M. Accuracy of an International Classification of Diseases Code Surveillance System in the identification of traumatic brain injury. Neuroepidemiology 2016;47:46-52.

61. Centers for Disease Control and Prevention. Glasgow Coma Scale. Available from: https://www.cdc.gov/ masstrauma/resources/gcs.pdf. Accessed 20 Sep 2021.

62. American College of Surgeons. Best practices in the management of traumatic brain injury. Available from: https://www.facs.org/-/media/files/quality-programs/ trauma/tqip/tbi_guidelines.ashx. Accessed 20 Sep 2021.

63. Carroll LJ, Cassidy JD, Holm L, Kraus J, Coronado VG, WHO Collaborating Centre Task Force on Mild Traumatic Brain Injury. Methodological issues and research recommendations for mild traumatic brain injury: the WHO Collaborating Centre Task Force on Mild Traumatic Brain Injury. J Rehabil Med 2004;(43 Suppl):113-25.

64. Kay T, Harrington DE, Adams R, et al. Definition of mild traumatic brain injury. J Head Trauma Rehabil 1993;8:867.

65. Kean J, Malec JF. Towards a better measure of brain injury outcome: new measures or a new metric? Arch Phys Med Rehabil 2014;95:1225-8.

66. McCrory P, Meeuwisse WH, Aubry M, et al. Consensus statement on concussion in sport: the 4th International Conference on Concussion in Sport, Zurich, November 2012. J Athl Train 2013;48:554-75. 